\title{
Fatores que influenciam a prática de exercícios resistidos em idosos no município de Patos-PB
}

\section{Factors that influence the exercise practice in the elderly in weathered county of Patos-PB}

\author{
Kaaio Rodrigo Santos Bezerra \\ Educador Físico formado pelas Faculdades Integradas de Patos-FIP. E-mail: kaaiorodrigo@outlook.com \\ José Maurício de Figueiredo Júnior \\ Docente das Faculdades Integradas de Patos-FIP. E-mail: mauricio22jr@hotmail.com \\ Juliana Almeida Leandro \\ Acadêmica do Curso de Educação Física das Faculdades Integradas de Patos-FIP. \\ E-mail: bezerra.almeida.jualmeida@ hotmail.com \\ Anne Milane Formiga Bezerra \\ Docente das Faculdades Integradas de Patos-FIP. E-mail: annemilane_pb@ hotmail.com \\ Kévia Katiúcia Santo Bezerra \\ Docente da Universidade Federal de Campina Grande-UFCG campus Cajazeiras-PB. \\ E-mail: keviabezerra@ hotmail.com
}

\begin{abstract}
Resumo: O processo de transição demográfica e de transição epidemiológica, ocorridos no Brasil e no mundo, fez com que aumentasse significativamente o número de idosos na sociedade, sendo o estilo de vida um fator importante na manutenção da saúde desses indivíduos. Assim, o surgimento de novas tecnologias na sociedade moderna, tornaram as pessoas cada dia mais sedentárias. As principais alterações fisiológicas ocorridas no envelhecimento geralmente estão intimamente ligadas e associadas a abusos de longo prazo na nutrição, fumo, atividades físicas inadequados e a exposição a agentes nocivos. Assim, objetivou-se analisar os fatores motivacionais para a prática de exercícios físicos resistidos em indivíduos idosos, identificando as principais dificuldades para adesão à prática de exercícios físicos e os possíveis benefícios ocasionados a partir dessa adesão. Trata-se de um estudo descritivo, quantitativo, que teve como amostra 22 idosos de ambos os gêneros na faixa etária entre 60 e 81 anos, matriculados na Academia Escola FipFitness. $\mathrm{O}$ instrumento utilizado para a coleta de dados foi um questionário sócio-demográfico e uma entrevista semi estruturada. Os resultados mostram que os fatores que mais influenciam os idosos a buscar a prática de atividades físicas são a melhora na qualidade de vida, a companhia dos amigos, ou seja, fator social e interferência familiar; o que resulta em uma manutenção destas atividades ao passo em que os mesmo percebem uma melhora em seu bem estar físico e psicológico.
\end{abstract}

Palavras-chave: Atividade física. Envelhecimento. Qualidade de vida

\begin{abstract}
The process of demographic transition and epidemiological transition that occurred in Brazil and in the world, caused significantly increase the number of older people in society, and the lifestyle an important factor in maintaining the health of these individuals. Thus, the emergence of new technologies in modern society, have made people increasingly sedentary day. The main physiological changes in the aging are often closely linked and associated with long-term abuse in nutrition, smoking, inadequate physical activity and exposure to harmful agents. The objective was to analyze the motivational factors for the practice of resistance exercise in elderly individuals, identifying the main difficulties for adherence to physical exercise and the possible benefits incurred from that membership. This is a descriptive, quantitative study, which was to sample 22 elderly of both genders aged between 60 and 81 years, enrolled in the Academy School Fip-Fitness. The instrument used for data collection was a socio-demographic questionnaire and a semi-structured interview. The results show that the factors that most influence the elderly to seek physical activity are the improvement in quality of life, the company of friends, or social factor and family interference; which results in a continuation of these activities while at the same notice an improvement in your physical well being and psychological.
\end{abstract}

Keywords: Physical activity. Ging. Quality of life 


\section{INTRODUÇÃO}

O processo de transição demográfica e de transição epidemiológica, ocorridos no Brasil e no mundo, fez com que aumentasse significativamente o número de idosos na sociedade, evidenciando estados peculiares de morbidade relacionados com as doenças crônicas, sendo estas, em grande parte, influenciadas pelo estilo de vida.

Por sua vez, a associação de novas tecnologias na sociedade moderna tornou as pessoas a cada dia mais sedentárias, fazendo com que haja um grande número de indivíduos com idade avançada sofrendo de alguma doença crônica. Projeta-se para o Brasil no ano de 2025, a sexta posição entre os países com o maior número de pessoas idosas. Essa posição vem seguida de dados alarmantes entre esta população (CÂMARA et al. 2006)Segundo Corazza (2001), a atividade física proporciona à terceira idade inúmeros benefícios dentre os quais podemos destacar; obtenção de saúde, domínio corporal, aumento da mobilidade, cura contra a depressão, uma respiração saudável, vitalidade, autoconfiança, reações positivas, tensão e ansiedades reduzidas, previne doenças (cardiorrespiratórias, hipertensão, diabetes e outras), e consequentemente promove a socialização e a melhora da capacidade funcional.

É notório que a prática da atividade física trás grandes benefícios para o nosso corpo, e, seja qual for à atividade deve ser estimulada com o objetivo de prevenir o aparecimento de doenças que possam comprometer a qualidade de vida do homem. Dessa forma promover o conhecimento da população em geral sobre os benefícios da atividade física é um importante passo, principalmente em indivíduos com idade superior aos 60 anos, mostrando-lhes os benefícios desta atividade.

Sabe-se que a atividade física melhora a qualidade de vida do homem em vários pontos, prevenindo doenças, a atividade física estimula a secreção de hormônio que agem diretamente no bem-estar do ser humano. Assim o presente estudo tem como objetivo analisar os fatores que influenciam para a prática de exercícios físicos resistidos em idosos.

\section{METODOLOGIA}

O presente estudo caracteriza-se como um estudo descritivo, o mesmo foi desenvolvido mediante uma abordagem quantitativa em relação aos idosos que praticam exercícios resistidos na Academia Escola das Faculdades Integradas de Patos - FIP. A amostra foi composta por 22 idosos, no qual a seleção da amostra se deu com base nos seguintes critérios de inclusão: está matriculado na Academia Escola FIP Fitness; ter no mínimo 3 (três) meses consecutivos de matrícula; ter acima de 60 anos de idade. $O$ instrumento de coleta de dados foi um questionário sócio-demográfico e em uma entrevista semiestruturada sobre os fatores influenciadores para a prática de exercícios resistidos em idosos, sendo esse baseado em Saba (1999). Os dados foram coletados, em um ambiente calmo e agradável, e sem interferências, afim de que o estudo não sofra intervenções, mediante aceitação voluntária no estudo. Diante de informações acerca dos objetivos do trabalho, aqueles que aceitaram participar do estudo foram convidados a assinar o Termo de Consentimento Livre e esclarecido - TCLE. Após a realização das entrevistas a análise dos dados ocorreu com base na interpretação das respostas obtidas utilizando-se da técnica de análise de conteúdo, bem como comparação dos achados com a literatura pertinente sobre o assunto em questão. A pesquisa foi realizada em conformidade com as considerações da Resolução No 466/2012 do Conselho Nacional de Saúde, que regulamenta a pesquisa envolvendo seres humanos. O projeto de pesquisa foi submetido ao Comitê de Ética das Faculdades Integradas de Patos e aprovado sob protocolo 051/2012. Para tanto os sujeitos da pesquisa foram assegurados do total sigilo sobre sua identidade e respostas confidenciais bem como a liberdade para participar ou desistir da pesquisa a qualquer momento sem que haja nenhum constrangimento (BRASIL, 2012).

\section{RESULTADOS E DISCUSSÃO}

Observa-se na tabela 01 , a caracterização sócio demográfica da amostra, onde o sexo dos idosos entrevistados 22,72 \% (05) da amostra são masculino e $77,27 \%$ (17) são do sexo feminino. Segundo estudo de Colares et al. (2009) as mulheres em sua maioria apresentam tendências e práticas relacionadas a baixas condutas de risco, demonstrando que esta grande diferença e afastamento considerável em relação a estética, tendências e moda é parte constante no universo feminino, sendo compartilhada em sua minoria também pelos homens.

Em relação a faixa etária dos entrevistados observa-se que idosos entre 60 a 65 anos, representam $36,36 \%$ (08), de 66 a 70 anos de idade são 22,72\% (05), de 71 a 75 anos representam 27,27\% (06), e de 76 a 81 representam $13,63 \%$ (03) idosos.

Segundo Barbosa (2001), a expectativa média de vida vem sofrendo um acréscimo, bem como a preocupação com uma melhor qualidade de vida e a satisfação harmoniosa dos objetivos e desejos que implicam numa idéia de felicidade. Além da procura e constante controle para a ausência de aspectos e sentimentos negativos.

Quanto ao estado civil a maior parcela dos idosos são casados, $50 \%$, evidenciado uma situação de estrutura familiar e vida conjugal. No que concerne a ocupação profissional, a pesquisa constatou que $86,37 \%$ (19) dos participantes continuam trabalhando e $13,63 \%$ são pensionistas do Instituto Nacional do Seguro Social (INSS) ou aposentados.

O predomínio de idosos inseridos no mercado de trabalho pode ter relação com proporção elevada de idosos que enfrentam dificuldades em adquirir aposentadoria, como também à melhora da expectativa de vida, nível de autonomia e vitalidade encontrada nos idosos da sociedade atual. A prática regular de atividade física também implica em fator positivo para continuidade da vida produtiva (SILVA; CUNHA, 2010). 
Tabela 1 - Caracterização da amostra segundo os dados sócios demográficos de idosos que praticam exercícios físicos na Academia Escola da FIP (Faculdades Integradas de Patos)

\begin{tabular}{|c|c|c|c|}
\hline Variáveis & Especificações & $\mathbf{f}$ & $\%$ \\
\hline \multirow[t]{2}{*}{ Sexo } & Masculino & 05 & 22.72 \\
\hline & Feminino & 17 & 77.27 \\
\hline \multirow[t]{4}{*}{ Idade } & $60-65$ anos & 08 & 36.36 \\
\hline & $66-70$ anos & 05 & 22.72 \\
\hline & $71-75$ anos & 06 & 27.27 \\
\hline & 76-81 anos & 03 & 13.63 \\
\hline \multirow[t]{3}{*}{ Atividade física que pratica } & Ginástica & 14 & 63.36 \\
\hline & Musculação & 07 & 31.81 \\
\hline & Dança & 01 & 4.54 \\
\hline \multirow[t]{2}{*}{ Turno que frequenta } & Manhã & 21 & 95.45 \\
\hline & Tarde & 01 & 4.54 \\
\hline Tempo que dura a atividade física & Mais de 1 hora & 22 & 100 \\
\hline \multirow[t]{6}{*}{ Escolaridade } & Não alfabetizado & 03 & 13.63 \\
\hline & Alfabetizado & 02 & 9.09 \\
\hline & Ensino Médio incompleto & 01 & 4.54 \\
\hline & Ensino Médio completo & 08 & 36.36 \\
\hline & Ensino Superior incompleto & 02 & 9.09 \\
\hline & Ensino Superior completo & 06 & 27.27 \\
\hline \multirow[t]{5}{*}{ Estado civil } & Solteiro & 1 & 4.54 \\
\hline & Casado & 11 & 50 \\
\hline & Viúvo & 5 & 22.72 \\
\hline & Divorciado & 5 & 22.72 \\
\hline & União Estável & 0 & \\
\hline \multirow[t]{6}{*}{ Horas de trabalho } & 4 horas & 1 & 4.54 \\
\hline & 5 horas & 1 & 4.54 \\
\hline & 6 horas & 3 & 13.63 \\
\hline & 8 horas & 12 & 54.54 \\
\hline & 10 horas & 2 & 9.09 \\
\hline & Não trabalha & 3 & 13.63 \\
\hline Total & & 22 & 100 \\
\hline
\end{tabular}

Em relação ao índice de escolaridade, os analfabetos representam 13,63\% (03), os alfabetizados somam 9,09\% (02), com ensino médio incompleto 4,54\% (01), com ensino médio completo $36,36 \%$ (08), superior incompleto $9,09 \%$ (02), ensino superior completo 27,27 (06), o que evidencia que a grande maioria sabe ler e escrever $86,37 \%$, mostrando assim um grande avanço no que diz respeito ao nível de instrução de idosos e contrastando com pesquisas mostram predomínio de baixo nível de instrução em idosos, como por exemplo, o estudo de Silva e Cunha (2010). Nesses casos associa-se a baixa instrução ao fato de que trata-se de indivíduos que viveram sua infância em épocas em que o ensino, ainda, não era prioridade, principalmente com relação à mulher, associado também a discriminação de gênero e à inexistência de políticas publicas de educação.

Observa-se na tabela 1 que $100 \%$ (22), dos entrevistados afirmaram que realizam uma hora de exercícios físicos diários de intensidade moderada a vigorosa na maioria dos dias da semana dependendo do protocolo elaborado pelo Profissional de educação. A intensidade vigorosa pode promover benefícios adicionais à intensidade moderada, mas também se reconhecem seus benefícios. Sobre o tipo de exercício físico realizado por esses idosos, os mesmos praticam ginástica com representação de $63,63 \%$ (14) idosos, musculação em $31,81 \%$ (07) dos idosos e dança aparecendo em $4,54 \%$ (01) dos entrevistados.
Relacionado ao principal objetivo do estudo, que busca identificar os aspectos motivacionais para prática de exercícios físicos em idosos, o estudo mostra que os principais motivos por ordem de frequência das respostas são: melhoria na qualidade de vida com 95,45\% (21), quando perguntados sobre a motivação á adesão de práticas de exercícios físicos, influência dos amigos representa $40,90 \%$ quando perguntados por qual motivo a escolha da academia e família com 22,72\% (05), aos expressarem a opinião em relação a influencia á pratica de exercícios físicos resistidos em academias, no entanto, os piores resultados ficaram por conta da estética com $0,0 \%$ seguida pelo fato de a academia se localizar perto de casa com $18,18 \%$ e por último por influencia da mídia também sem ter apresentado pontuação, respectivamente nas perguntas acima explanadas (Figura 01).

Em contrapartida, quando indagados sobre as principais dificuldades para adesão à programas de exercício físico, o estudo mostrou que em sua grande maioria os idosos relatam não haver motivo aparente $(64,7 \%)$, seguido do próprio comodismo $(23,52 \%)$ e disponibilidade de tempo relatado em $11,76 \%$ dos idosos estudados. 
Figura 1- Caracterização da amostra de acordo com os aspectos motivacionais para prática de exercícios físicos em idosos

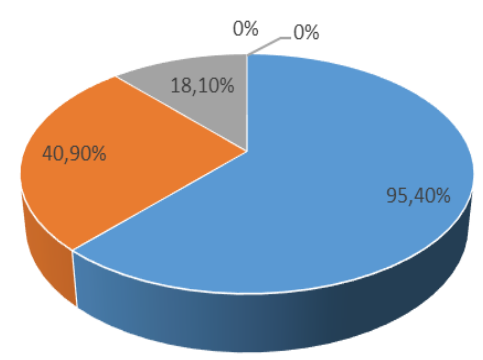

$$
\begin{aligned}
& \text { " Melhoria da qualidade de vida - Influência dos amigos } \\
& \text { " Academia perto de casa = Estética } \\
& \text { - Influencia da mídia }
\end{aligned}
$$

É necessário o uso de diferentes estratégias, incluindo a informação e convencimento dos idosos de que a prática de exercícios resistidos irá interferir de forma benéfica, na qualidade de vida dos mesmos.

Segundo Saba (2001), a disponibilidade para a prática de exercícios físicos, em comunhão com os dados verificados nesta pesquisa, se origina de um conjunto de determinantes pessoais, ambientais que propicia a manutenção da prática física por longos períodos de tempo, podendo ser entendida como a verdadeira legitimação e percepção do próprio idoso de que sua qualidade de vida melhorou, obtendo assim o ápice de uma evolução constante, rumo a prática do exercício físico.

Nonomura (1998) aponta de forma pertinente as dificuldades, bem como os fatores que poderiam ser considerados influenciadores para prática de exercícios físicos resistidos relatados pelos entrevistados nesta pesquisa, citando quatro possibilidades em relação a forma de encarar a prática de exercícios físicos, seriam adoção, manutenção, desligamento, retomada da atividade.

Tais dados demonstram uma crescente vontade e conscientização dos idosos quanto a importância da prática de atividades físicas regulares, na qual os indivíduos participantes deste estudo referiram a percepção de alterações de ordem física ou psicológica, onde o alívio do estresse foi citado por $45,45 \%$ dos entrevistados, motivação em $27,27 \%$, seguidos por aumento da massa muscular, perda de peso e aqueles que não notaram diferença, representando $9,09 \%$ cada um.

É sabido que a velhice traz consigo um declínio natural nas capacidades físicas e funcionais, bem como perda de massa óssea e muscular, comprometimento da elasticidade e flexibilidade articularem, ganhos de peso e com isso maior lentidão de movimentos o que traz consigo o aparecimento de doenças crônicas (CARVALHO; NETO, 1994). Os benefícios da prática de atividades físicas, independente da forma, seja ginástica, musculação ou dança, contribui para a melhora na saúde e conseqüentemente aumenta a longevidade, evitando a incapacidade associada ao envelhecimento.

\section{CONCLUSÕES}

A procura de uma melhor qualidade de vida, bem como a possibilidade de socializar-se são definidos na forma de pensar deste grupo em que este estudo tomou como base, sendo importantes influenciadores para prática de exercícios resistidos.

\section{REFERÊNCIAS BIBLIOGRÁFICAS}

BARBOSA, J. H. P. Educação física em programas de saúde. In Curso de Extensão Universitária Educação Física na Saúde, 2001. Centro universitário ClaretianoCEUCLAR - Batatais, SP.

BRASIL, M. S. da. Conselho Nacional de Saúde. Comissão Nacional de Ética em Pesquisa- CONEP. Resolução $n^{\circ} 466 / 2012$ sobre pesquisa envolvendo seres humanos. Brasília, 2012.

CÂMARA， L. C， SANTAREM J.M， FILHO W.J, KUWAKINO M.H - Exercícios resistidos em idosos portadores de insuficiência arterial periférica. Acta Fisiatr, v.13, n.2, p.96-102,2006

CARVALHO F.; NETO, M. P.: Geriatria: fundamentos, clínica e terapêutica. Rio de Janeiro: Atheneu, 1994.

CAROMANO.bF. A. CANDELORO.J.M. Fundamentos da hidroterapia para idosos. Arq. Cienc. Saúde Unipar. 2001. 5(2); 187-95

COLARES V., FRANCA C., GONZALEZ E. Condutas de saúde entre universitários: diferenças entre gêneros. Cadernos de Saúde Pública. V.25, n.3, 2009.

CORAZZA. M. A. Terceira idade e atividade física. 1. Ed, São Paulo: Phorte, 2001.

GATTO. I.B. Aspectos psicológicos do envelhecimento. IN: PAPALEO NETTO. M. Gerontologia: a velhice e o envelhecimento em visão globalizada. São Paulo: Atheneu, 2002.

NONOMURA, M. Motivos de adesão à atividade física em função das variáveis idade, sexo, grau de instrução e tempo de permanência. Revista Brasileira de Atividade Física e Saúde, v. 3, n. 3, p. 45-58, 1998.

PAPALÉO NETTO. M. Tratado de gerontologia. São Paulo: Atheneu: 2007.

SABA, F. Aderência: à prática do exercício físico em academias. São Paulo: Manole, 2001.

SABA, F. Determinantes da prática de exercício físico em academias de ginástica. Dissertação (Mestrado). Escola de Educação Física e Esporte, Universidade de São Paulo, São Paulo, 1999. Disponível em : http://www.nuteses.temp.ufu.br/tde_busca/arquivo.php?co dArquivo=3953\&acordo. Acesso em: 07 ago de 2010.

SILVA, R. R.; CUNHA, G. A importância da prática da atividade física em indivíduos idosos. Portal revistas. ucb. BR/ índex. 2010. Disponível em: https://portalrevistas.ucb.br/index.php/efr/article/view/154 7/1130. Acesso em: 06 ago de 2010. 